19 Revue d'histoire du XIXe siècle

Société d'histoire de la révolution de 1848 et des

révolutions du XIXe siècle

25 | 2002

Le temps et les historiens

\title{
Conclusion.Le temps des historiens ou regards sur le passé
}

Jean-Claude Caron

\section{OpenEdition \\ Journals}

Édition électronique

URL : http://journals.openedition.org/rh19/421

DOI : $10.4000 /$ rh19.421

ISSN : $1777-5329$

Éditeur

La Société de 1848

Édition imprimée

Date de publication : 1 décembre 2002

Pagination : 199-202

ISSN : 1265-1354

Référence électronique

Jean-Claude Caron, "Conclusion.Le temps des historiens ou regards sur le passé », Revue d'histoire du XIXe siècle [En ligne], 25 | 2002, mis en ligne le 29 juin 2005, consulté le 30 avril 2019. URL : http:// journals.openedition.org/rh19/421 ; DOI : 10.4000/rh19.421

Ce document a été généré automatiquement le 30 avril 2019

Tous droits réservés 


\title{
Conclusion.Le temps des historiens ou regards sur le passé
}

\author{
Jean-Claude Caron
}

1 Que retenir, que synthétiser à partir des différentes communications présentées lors de cette journée? Tout d'abord des évidences, mais qu'il semble nécessaire de rappeler. Ainsi que le temps est le matériau premier de l'historien. Il est même, au fond, le seul matériau sur lequel il puisse prétendre réfléchir (au sens de penser, mais aussi au sens de renvoyer une image, comme un miroir), dans la mesure où l'histoire n'a de sens que parce qu'elle envisage l'action des hommes non d'un point de vue philosophique, psychologique ou fictionnel, mais d'un point de vue chronologique. On a pu dire que le présent n'existait pas, ou que seule l'expérience qui incarnerait le passé et l'espoir qui incarnerait le futur en tiendraient lieu, dans une tension toujours vive. Ce qui revient à dire, aussi, qu'il n'y a pas de temps sans chronologisation, sinon, il s'agirait d'un temps en quelque sorte intemporel, donc imperceptible. Précisons qu'il ne s'agit pas de limiter et encore moins d'assimiler l'histoire à la seule chronologie, mais plus simplement de constater que la légitimité de la discipline histoire vient du fait qu'elle est née dans cette volonté à la fois simple et orgueilleuse de dire ce qui est advenu, par rapport à ce qui est devenu, de décrire l'avènement du devenir des hommes. La formule paraîtra redondante et banale, mais le passé, c'est ce qui s'est passé et que l'historien doit passer : il est une sorte de passeur du passé.

2 L'objet-temps est nécessairement disciplinaire : chaque discipline a construit son rapport au temps et même s'est construite par rapport au temps. Le temps des mathématiciens ou des biologistes n'est pas le temps des historiens. Dès lors, le partage du concept de temps se révèle difficile : ne constitue-t-il pas, en définitive, l'obstacle le plus redoutable, car le moins perceptible, de la communication entre disciplines? Même les disciplines les plus voisines de l'histoire, comme la philosophie ou la sociologie, n'envisagent pas le temps comme le font les historiens --et inversement. L'historien produit et utilise essentiellement deux usages du temps : on pourra les nommer écart et discontinuité. Le temps des historiens, c'est d'abord la prise en compte de l'écart qui le sépare, lui, le sujet vivant dans un temps que l'on pourrait appeler actuel, de préférence à présent, du temps 
de l'objet. Mais l'historien doit aussi prendre en compte le fait que des discontinuités temporelles s'établissent entre les acteurs de l'histoire. La première difficulté consiste donc à situer les acteurs de l'histoire dans leur temporalité : difficulté double, car d'une part, tous les acteurs de l'histoire ne s'inscrivent pas dans la même temporalité, ou, pour dire les choses plus simplement, n'ont pas le même rapport au temps (de même qu'ils n'entretiennent pas le même rapport à l'espace); et, d'autre part, tous les acteurs de l'histoire n'ayant pas un accès direct à la parole, ce sont d'autres acteurs qui parlent en leur nom, et leur attribuent leur propre rapport au temps, le temps dominant. Cette question des temporalités divergentes est fondamentale, en ce qu'elle conditionne l'écriture même de l'histoire, c'est-à-dire le passage au récit qui est d'abord et avant tout un ordonnancement du temps. La contrainte est donc double : celle de penser le passé au présent se conjugue avec celle de penser le futur du présent. L'écriture de l'histoire est projection d'un advenu dans un devenir. En ce sens, il y a bien passage par la fiction, c'està-dire par l'utilisation de temporalités construites pour les besoins de la démonstration historique.

3 Dans un compte-rendu du congrès international des sciences historiques d'Oslo, Roger Chartier a affirmé que l'un des principaux dangers qui guettait les historiens, surtout ceux du temps très contemporain, était l'anachronisme. L'affirmation mérite d'être discutée : car elle sous-tend l'idée que l'historien pourrait objectivement penser l'écart entre le présent et le passé, et même dépasser cet écart en évitant de penser le passé avec les concepts du présent, mais aussi qu'il pourrait toucher à "la" réalité, voire à "la" vérité du passé, en bref qu'il y aurait un "passé vrai" que l'abolition de l'écart au présent rendrait perceptible dans une interprétation une et unique. Mais comment penser l'écart entre présent et passé, dans la mesure où la seule compréhension que nous avons du temps relève de l'expérience et non de la pensée ? De même, pourquoi lutter contre la connaissance de ce qui advient ? On se situe alors, pour le coup, dans la négation de ce qui constitue l'écart entre l'événement et nous. Faire "comme si" on ne connaissait pas la suite du fil du temps ne peut être réellement possible : c'est un artifice encore plus lourd, un handicap encore plus pesant que celui qui est parfois dénoncé comme la "tyrannie du passé". Il n'existe pas d'écriture de l'histoire qui puisse évacuer l'après, ni l'écart, dans la connaissance de l'avant. Si l'on admet que l'histoire, dans son rapport au temps, obéit à la règle de la combinaison des possibles, alors la connaissance de l'après et l'anachronisme ne deviennent plus des adversaires, mais des alliés. Comme l'énonce fort simplement Paul Ricœur, "le passé a eu lieu". Dès lors, nous proposons d'employer deux autres termes permettant d'éviter le recours à celui d'anachronisme: celui d'hétérochronisme, pour désigner les divergences temporelles ou temporalités hétérogènes entre les acteurs de l'histoire; et celui d'homochronisme pour désigner les convergences temporelles ou temporalités convergentes entre ces mêmes acteurs. L'écriture de l'histoire est une perpétuelle restitution, mais aussi une perpétuelle conciliation entre le temps et la mémoire, qu'un conflit structurel oppose en permanence : la résolution de la tension entre les deux est le propre même du travail de l'historien.

4 Mais il convient aussi de s'interroger sur la question des convergences et des divergences temporelles entre les producteurs de l'histoire que sont les historiens : sommes-nous sûrs de partir de la même expérience temporelle, de la même perception ou du même rapport au temps, qu'il soit passé, présent ou futur? Penser le temps, c'est le penser en fonction de notre expérience; c'est un acte individuel où interviennent, au milieu de données partagées, des conditions propres à chacun d'entre nous. L'écriture de l'histoire, en ce 
qu'elle est d'abord et avant tout le rapport à son temps propre, ou le reflet d'une individuation temporelle, même si on ne peut nier que l'on s'inscrit, volens nolens, dans un temps collectif, apparaît comme le révélateur d'une hétérochronie constitutive même de la conscience humaine. On n'échappe jamais vraiment au temps qui constitue sa propre histoire et qui, sous la forme de la mémoire, habite, nourrit et parfois encombre notre inconscient, telle une sélection d'événements dont certains, pourtant chronologiquement très proches, nous semblent très lointains, et inversement. Il ne s'agit pas de prôner une histoire psychologique qui nierait l'idée même que l'on puisse "partager" le temps, mais de constater que, dans la construction du temps que nous opérons nécessairement, de façon consciente ou non, dans la définition de notre objet historique, nous projetons une temporalité personnelle qui, face au(x) discours constituant notre source, nous fait mettre l'accent sur tel mot et donc sur telle chose, plutôt que sur tel ou telle autre. Ce refoulé que nous traquons dans la source, et qui a précisément pour objet le passé, médiat ou immédiat, est également présent dans notre histoire, faisant écran, parfois, à ce que nous ne voulons pas voir : parce que l'oubli, le refoulement ou la réécriture du passé sont des conditions nécessaires à l'acceptation du présent et à la projection dans le futur. Le “je" de l'historien est présent à tous les stades du processus de l'écriture de l'histoire: depuis l'inscription du sujet choisi dans une temporalité jusqu'à la production du récit -peut-on parler, au sujet de la construction de ce dernier, du temps des mots?

De même que nous --vivant dans le temps au présent-- ne pouvons échapper à la contingence du temps, l'histoire n'échappe pas à la tyrannie du temps; mais gardonsnous, pour autant, de pré-établir le temps de l'histoire, tâchons de le construire en fonction de l'objet qui est le nôtre. Car le temps est une construction au service d'une démonstration. Alors que la durée est l'expression d'une réalité objective, capable d'être inscrite dans une chronologie du réel, universelle car participant d'une même acceptation du comptage temporel, le temps est un système élaboré pour donner un sens à une somme de durées, dans une perspective à la fois causale (l'avant) et conséquentielle (l'après). Le temps est la perception de la durée selon notre expérience. D'où le fait que l'écriture de l'histoire se déroule, pour employer une métaphore musicale, sur une partition dont les notes --blanches, noires ou croches-- n'auraient pas forcément la même durée pour tous. En résumé, le temps est un mode d'explication d'un présent --incluant le temps qui est au cœur d'une recherche historique et qui appartient pourtant au passé-défini par un avant et un après. Le temps produit le décalage, mieux : est le produit du décalage avec la durée, car attribuant des valeurs temporelles différentes suivant les événements, à la fois dans leur perception immédiate par les contemporains et dans leur temporalisation par les historiens : un jour d'insurrection ou de révolution est plus qu'un jour, il est extrait de l'échelle de la durée pour passer à l'échelle du temps, et sa durée réelle est prolongée, étendue par une temporalité historique qui en permet la compréhension jusqu'à nos jours, en tout cas l'actualise et en fait un objet d'histoire. Ce qui revient à dire que le passé n'est pas un objet fixe, mais est ce que nous en faisons, ce par quoi et pourquoi nous décidons qu'il est, en fonction de lectures multiples, mais aussi d'usages différents de ce passé. L'historien construit un ordre du temps, rarement défini (on est dans le règne de l'implicite, le plus souvent), et jamais définitif. L'ordonnancement du passé qu'est l'écriture de l'histoire s'inscrit dans un temps en mouvement: mais ce n'est pas nous, dans un présent mouvant, qui allons vers un passé fixe, mais l'inverse.

Jean-Claude Caron est professeur à l'université de Clermont-Ferrand 


\section{RÉSUMÉS}

Le temps des historiens est bâti sur les notions d'écart et de discontinuité. Mais l'historien doit aussi prendre en compte l'existence de temporalités différentes. Plus que la notion d'anachronisme, nous proposons d'utiliser les notions d'hétérochronisme et d'homochronisme. L'historien doit intégrer sa propre temporalité dans son rapport au temps, et être conscient qu'il construit un ordre du temps en fonction de son objet.

The time of historians --or how to look on the past

The time of historians is built on two notions: gap and discontinuity. But the historian must also take into account the existence of different temporalities. More than the notion of anachronism, we suggest to use the notions of heterochronism and homochronism. The historian has to integrate his own temporality in his / her relationship with the notion of time and must be aware that he / she builds an order of time according to the subject of his / her research. 